ARTICLE

\title{
Target-responsive vasoactive probes for ultrasensitive molecular imaging
}

\author{
Robert Ohlendorf (1,5, Agata Wiśniowska ${ }^{2,5}$, Mitul Desai ${ }^{1}$, Ali Barandov ${ }^{1}$, Adrian L. Slusarczyk ${ }^{1}, \mathrm{Nan}_{\mathrm{Li}}{ }^{1} \&$
} Alan Jasanoff $\mathbb{1 D}^{1,3,4 凶}$

The ability to monitor molecules volumetrically throughout the body could provide valuable biomarkers for studies of healthy function and disease, but noninvasive detection of molecular targets in living subjects often suffers from poor sensitivity or selectivity. Here we describe a family of potent imaging probes that can be activated by molecules of interest in deep tissue, providing a basis for mapping nanomolar-scale analytes without the radiation or heavy metal content associated with traditional molecular imaging agents. The probes are reversibly caged vasodilators that induce responses detectable by hemodynamic imaging; they are constructed by combining vasoactive peptides with synthetic chemical appendages and protein blocking domains. We use this architecture to create ultrasensitive biotinresponsive imaging agents, which we apply for wide-field mapping of targets in rat brains using functional magnetic resonance imaging. We also adapt the sensor design for detecting the neurotransmitter dopamine, illustrating versatility of this approach for addressing biologically important molecules.

\footnotetext{
${ }^{1}$ Department of Biological Engineering, Massachusetts Institute of Technology, 77 Massachusetts Ave. Rm. 16-561, Cambridge, MA 02139, USA. ${ }^{2}$ HarvardMIT Health Sciences \& Technology, Massachusetts Institute of Technology, 77 Massachusetts Ave. Rm. 16-561, Cambridge, MA 02139, USA. ${ }^{3}$ Department of Brain \& Cognitive Sciences, Massachusetts Institute of Technology, 77 Massachusetts Ave. Rm. 16-561, Cambridge, MA 02139, USA. ${ }^{4}$ Department of Nuclear Science \& Engineering, Massachusetts Institute of Technology, 77 Massachusetts Ave. Rm. 16-561, Cambridge, MA 02139, USA. ${ }^{5}$ These authors contributed equally: Robert Ohlendorf; Agata Wiśniowska. ${ }^{凶}$ email: jasanoff@mit.edu
} 
M apping molecular species with minimal invasiveness in whole living organisms could provide insight into diverse physiological functions and their disruption in disease. Fluorescent sensors are commonly used to monitor molecular processes at high resolution in vivo ${ }^{1,2}$, but detection of fluorescent probes in most organisms is limited by optical scattering and absorption to superficial and often decontextualized regions of the body ${ }^{3-6}$. Sensitive detection of probes at subnanomolar concentrations in deep tissue is possible using nuclear imaging, but analyte-responsive probes for nuclear techniques are not available, so these methods can only measure localization and kinetics of the tracers themselves ${ }^{7}$. Magnetic resonance imaging (MRI) contrast agents are detectable in deep tissue, and can be sensitized to a variety of biologically relevant targets ${ }^{8}$, but imaging agents for MRI are usually only detectable at micromolar concentrations, or at similar mass doses when nanoparticle contrast agents are used. This creates challenges for delivery of the probes, increases the potential for physiological disruptions or toxicity, and often means that only high analyte levels can be detected.

Vasoactive probes (vasoprobes) are a recently developed class of imaging agent that can be detected at an organ- or organism-wide scale using diverse noninvasive imaging modalities, without the need for radioactive or metallic components ${ }^{9}$. Effective vasoprobes can be derived from potent vasodilatory peptides that induce relaxation of the vascular smooth muscle cells (VSMCs) that surround most blood vessels. This in turn leads to spatiotemporally localized changes in blood flow, volume, and oxygenation that produce contrast changes in MRI, ultrasound, nuclear imaging, and optical imaging, analogous to the widely exploited hemodynamic bases of functional neuroimaging methods ${ }^{10-13}$. Recent results show that vasoprobes can produce measurable signals at nanomolar concentrations in the rodent brain and that both secretion and proteolytic uncaging of vasoprobes give rise to specific signatures in imaging? . Vasoprobes thus combine the versatility of paramagnetic MRI contrast agents with over 1000 times greater sensitivity to detection in imaging 8 . We therefore reasoned that the vasoprobe contrast mechanism could be harnessed to create potent sensors for biologically important molecules, and that target-responsive vasoprobes could facilitate detection of molecular species at the nanomolar concentrations characteristic of many signaling molecules, biochemical markers, and therapeutic agents.

In this study, we present a vasoprobe-based strategy for sensing molecules by hemodynamic imaging in vivo. We engineer activatable vasoprobes for analyte targeting (AVATars) with the aid of a cell-based in vitro bioassay, and show that the resulting imaging probes permit target-responsive detection of nanomolarscale species in live rat brains. We use our first AVATar to map biotin derivatives in vivo, demonstrating noninvasive detection of dilute biotinylated epitopes in intact tissue. We then demonstrate the generality of our design by creating a second AVATar for detection of the neurotransmitter dopamine, indicating the potential of vasoprobe technology for addressing a wide variety of molecular phenomena in the brain and other organs.

\section{Results}

Platform for AVATar construction. We chose to derive AVATars from the potent vasodilator pituitary adenylate cyclaseactivating polypeptide (PACAP), a 38-residue peptide that activates $G$ protein-coupled receptor-dependent cyclic adenosine monophosphate (cAMP) signaling in VSMCs with half-maximal effective doses $\left(\mathrm{EC}_{50}\right)$ in the sub-nanomolar range ${ }^{14}$ (Fig. 1a). We envisioned an AVATar design wherein a PACAP derivative is labeled with a tethered version of the target molecule of interest, which in turn interacts with a protein domain that selectively binds either the tethered target or the target molecule itself, in competition (Fig. 1b). In the absence of the target molecule, the protein domain would remain bound to the PACAP moiety via the tethered ligand, blocking the PACAP moiety from activating its receptors. The presence of elevated concentrations of the target molecule in tissue, however, would cause release of the PACAP a

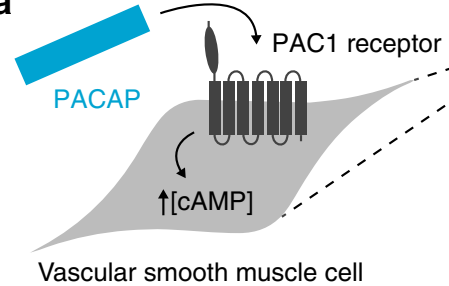

C

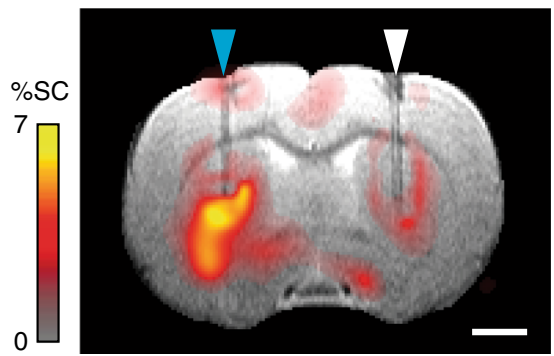

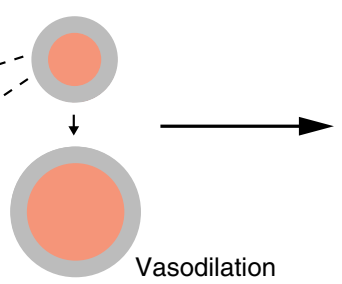

b

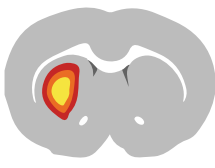

Hemodynamic imaging signal

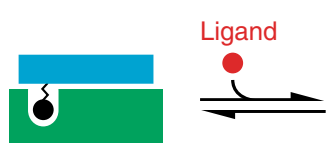

Low vasoactivity

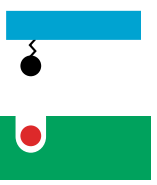

High vasoactivity
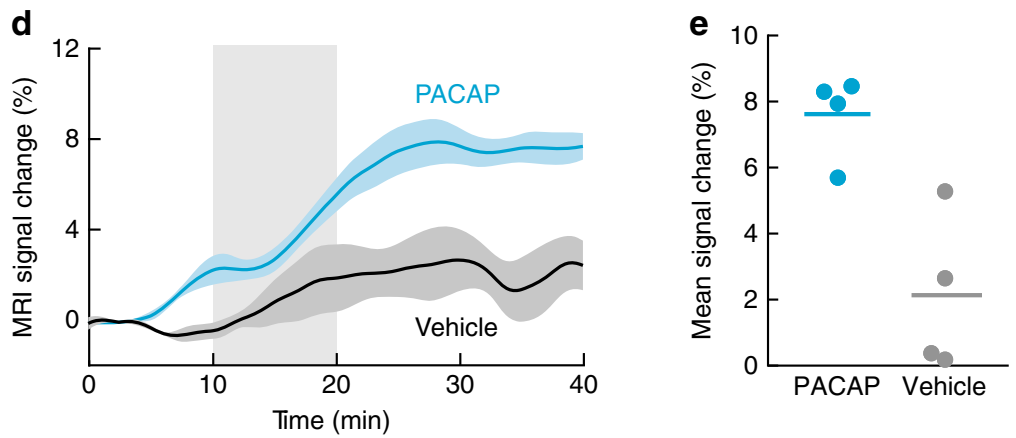

Fig. 1 Platform for AVATar construction. a PACAP (blue) functions as a vasoprobe by binding to receptors on vascular smooth muscle cells and activating a cAMP-dependent signaling cascade, causing blood vessel dilation and a hemodynamic imaging signal. $\mathbf{b}$ AVATar architecture: a tethered version of the target molecule is attached to PACAP peptide (blue); binding of a blocking domain (green) to the tethered target prevents receptor activation. Elevated free target molecule concentrations free active PACAP from the blocking interaction. c Map of mean MRI signal change following intracranial delivery of $1 \mu$ M PACAP (blue arrowhead) compared to aCSF vehicle (white arrowhead), overlaid on an anatomical scan. Scale bar $=2 \mathrm{~mm}$. $\mathbf{d}$ Mean MRI time courses during delivery of $1 \mu \mathrm{M}$ PACAP (blue) or aCSF vehicle (black). Shading represents SEM $(n=4)$. e Average MRI signal change $30-40$ min after PACAP or vehicle injection (horizontal lines $=$ mean values, $n=4$ ). 
derivative from the blocking protein, activating the AVATar and evoking a local hemodynamic imaging signal.

To verify that PACAP could be a suitable basis for constructing such sensors, we first examined whether this peptide could function as an effective vasoprobe in the living rat brain (Fig. 1c). Intracranial delivery of $1 \mu \mathrm{M}$ PACAP caused a mean hemodynamic blood oxygenation level-dependent (BOLD) signal change of $7.6 \pm 0.6 \%$ in $T_{2}{ }^{*}$ relaxation-weighted MRI, compared to only $2.1 \pm 1.2 \%$ in response to control injections of artificial cerebrospinal fluid (aCSF) vehicle (Fig. 1d, e). This difference is highly significant, with $t$-test $p=0.007(n=4)$; the signal change observed with the control injection probably arises from mild edema due to the introduction of fluid. Injection of $100 \mathrm{nM}$ PACAP also produces substantial hemodynamic responses compared with vehicle (Supplementary Fig. 1). Although we did not explore lower injection doses in vivo, published in vitro data suggests that the detection limit for PACAP concentrations in tissue is likely to be as low as $0.2 \mathrm{nM}^{14}$, but injection of doses calibrated to achieve intraparenchymal concentrations near this value is technically challenging. PACAP-dependent signals are repeatable over sequential blocks of injection (Supplementary Fig. 2), and produce responses comparable to BOLD signal changes evoked by somasensory stimulation (Supplementary Fig. 3). These results suggest that PACAP can indeed function as a robust platform for AVATar design.

Engineering a PACAP-based AVATar. We decided to apply the AVATar principle initially for sensing the small molecule biotin.
Because biotin is widely used as a labeling moiety, a vasoactive biotin sensor can be immediately useful as a means of detecting endogenously or exogenously biotin-labeled species in tissue, thus facilitating radiation-free in vivo analogs of powerful assays and histology methods ${ }^{15}$, as well as pretargeted imaging approaches ${ }^{16}$. With a molecular weight of $244 \mathrm{Da}$, biotin is similar in size and characteristics to many endogenous signaling molecules and metabolites, so we also anticipated that an imaging sensor for biotin could subsequently be adapted for detection of other targets. The tetrameric $53 \mathrm{kDa}$ protein streptavidin (SA) and its close relatives bind tightly to both free and functionalized biotin derivatives ${ }^{17}$, and are obvious candidates to act as blocking domains. The AVATar design also requires site-specific tethering of biotin to PACAP in a way that does not severely interfere with receptor activation, allows for binding of the blocking domain, and results in a sufficient activity change between blocked and unblocked states, such that substantial vasodilation occurs only in the unblocked state. We used a combination of structural data ${ }^{18}$ and previous structure-function analysis results ${ }^{14,18-20}$ to identify candidate biotin attachment sites likely to satisfy these criteria (Fig. 2a). A set of PACAP derivatives was then prepared, for which each of these sequence positions was replaced with a lysine-biotin residue.

To rapidly screen the modified peptide variants, we established a luminescence assay in mammalian cells that models PACAPrelated vasoactivity by quantitatively reporting cAMP production in response to activation of the PACAP receptor PAC1 (Fig. 2b, c). Titration of PACAP derivatives in this assay enabled $\mathrm{EC}_{50}$ values a

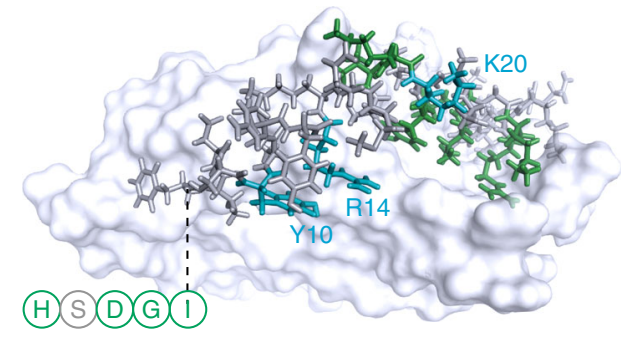

b

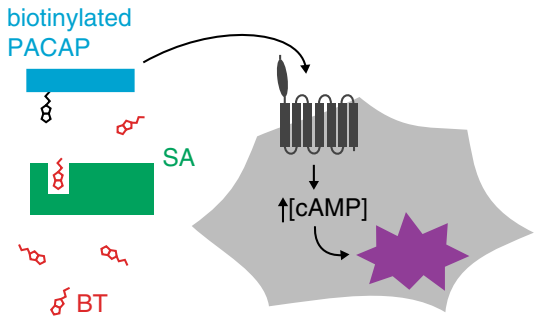

C

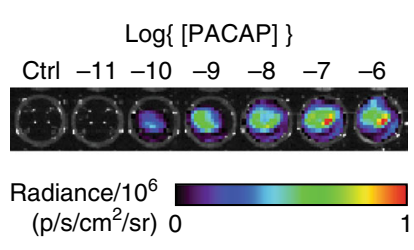

f

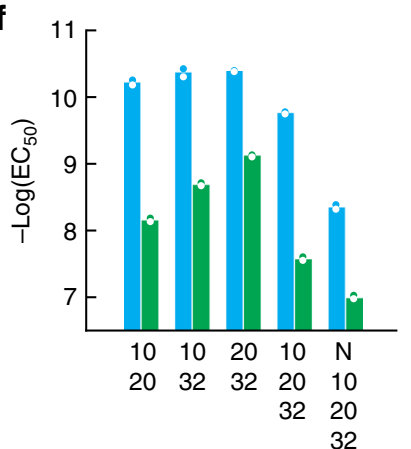

Fig. 2 Engineering a PACAP-based AVATar. a Identification of candidate ligand attachment sites on PACAP: Structure of PACAP in complex with a PAC1 receptor fragment ${ }^{18}$, highlighting residues whose substitution blocks (green) or does not affect (blue, labels) peptide bioactivity, according to previous structure-activity studies. N-terminal residues absent from the structure indicated schematically (circles). b Schematic of PAC1-based bioassay for testing biotinylated PACAP constructs (blue), their blocking by streptavidin (green), and release by free biotin (red). Unblocked PACAP derivatives can bind the $\mathrm{PAC1}$ receptor (dark gray) expressed in $\mathrm{CHO}$ cells, triggering CAMP production and activating a luminescence reporter (purple). c Raw luminescence recorded from the assay cells under titration with wild type PACAP. d Quantification of luminescence output and determination by curve-fitting of $\mathrm{EC}_{50}$ values for a biotinylated PACAP derivative (PACAP-10-BT) in the presence (green) and absence (blue) of $200 \mathrm{nM}$ streptavidin (SA). e Screening for PACAP residues that tolerate modification: $\mathrm{EC}_{50}$ values for receptor activation in the absence (blue) or presence (green) of SA by PACAP-biotin conjugates as a function of sequence position (untested positions in gray). $\mathrm{EC}_{50}$ of wild-type (WT) PACAP indicated by the dashed line. $\mathbf{f}$ EC ${ }_{50}$ values of multiply biotinylated PACAP conjugates modified at the positions noted ( $\mathrm{N}=\mathrm{N}$-terminus), in the absence (blue) and presence (green) of SA. Error bars in $\mathbf{d}$-e denote SD of duplicate measurements. 

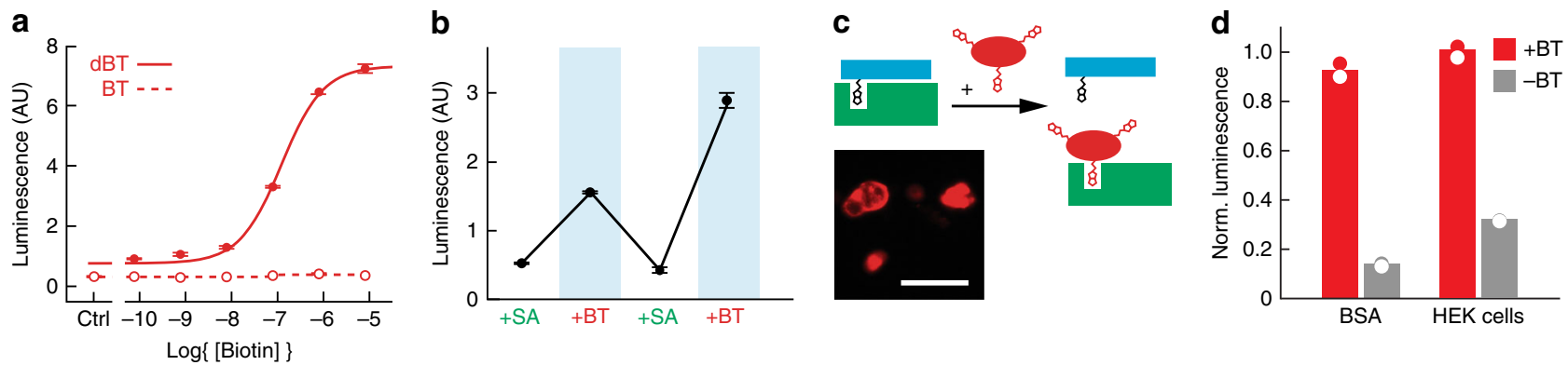

Fig. 3 Validation of BT-AVATar in vitro. a Titration of BT-AVATar containing dBT-modified PACAP (solid line) showing activation with $\mathrm{EC}_{50}$ of $120 \pm$ $10 \mathrm{nM}$. An equivalent variant of the sensor containing BT-modified PACAP (dashed line) is not activated by biotin. b Biotin vasosensor activity over repeated cycles of excess SA and biotin (BT) addition. c Schematic showing AVATar activation by biotinylated proteins or cells (red). Inlay shows biotinylated HEK cells stained with fluorescent streptavidin. Scale bar $=50 \mu \mathrm{m}$. Experiment was performed twice. d BT-AVATar is robustly activated by biotinylated bovine serum albumin and HEK cells (red) but not by unbiotinylated controls (gray). All error bars denote SD of duplicates.

to be obtained for each variant, in both the presence and absence of SA (Fig. 2d). With the exception of several residues near the Nterminus, biotinylation of most PACAP residues is well tolerated in the absence of $\mathrm{SA}$, with variants displaying $\mathrm{EC}_{50}$ values only modestly worse than the value of $10.8 \pm 0.2$ pM exhibited by wildtype PACAP (Fig. 2e). Loss of potency by $\sim 300$-fold upon biotinylation of positions $2-5$ is consistent with previous studies reporting the requirement for these residues in receptor activation $^{14,18}$. Of the PACAP derivatives evaluated, those with biotinylation of sites in the mid-region of the peptide produced the greatest SA-dependent dynamic range in receptor activation. In particular, a PACAP variant biotinylated at residue Y10 (PACAP-10-BT) provides the best combination of preserved bioactivity and sensitivity to SA binding, with $\mathrm{EC}_{50}$ values of $63 \pm$ $1 \mathrm{pM}$ and $1.24 \pm 0.05 \mathrm{nM}$ in the absence and presence of SA, respectively, corresponding to a 20 -fold change in potency (Fig. 2d). A PACAP variant with positions 10 and 20 simultaneously biotinylated (PACAP-10,20-BT) produced an even greater dynamic range, with an $\mathrm{EC}_{50}$ of $61 \pm 7 \mathrm{pM}$ that is reduced by a factor of $115 \pm 22$ in the presence of SA (Fig. 2f). Addition of further PACAP biotinylation sites did not substantially improve on this SA response, however, nor did modifications to the biotinpeptide linker length or repurification to remove contaminants from peptide synthesis (Supplementary Table 1).

Detecting biotin with nanomolar sensitivity in vitro. Based on these results, we designed a functional biotin-responsive sensor, BT-AVATar. The combination of PACAP-10,20-BT and SA could in principle function as a suitable sensor in itself, but the characteristic dissociation time of SA from biotin $(\sim 7 \mathrm{~h})^{21}$ is too long for meaningful applications based on the competition principle of Fig. 1b. To achieve effective biotin sensing, we therefore replaced the tethered biotin residues in PACAP-10,20BT with desthiobiotin, a similar compound that binds SA with 10,000 -fold lower affinity ${ }^{22}$ than biotin and can be easily displaced by it $^{23}$. The resulting peptide, PACAP-10,20-dBT, is not as effectively blocked by SA as PACAP-10,20-BT, but restoration of further modification sites addresses this problem (Supplementary Fig. 4). A variant in which tethered desthiobiotins were attached at positions 10, 20, and 32 proved optimal; this PACAP-10,20,32$\mathrm{dBT}$ displayed $\mathrm{EC}_{50}$ values of $130 \pm 2 \mathrm{pM}$ and $8.2 \pm 0.6 \mathrm{nM}$ in the absence and presence of SA, a robust 63-fold difference in potency. BT-AVATar was then formed by mixing $1 \mathrm{nM}$ PACAP10,20-32-dBT with two-fold molar excess of SA.

Titration of BT-AVATar with varying amounts of biotin in the PAC1 bioassay reveals an $\mathrm{EC}_{50}$ for biotin detection of $120 \pm 10 \mathrm{nM}$ (Fig. 3a). This is sensitive enough to detect typical biotinylation levels associated with biotin display or labeling technologies ${ }^{15}$, but not so sensitive as to be triggered by endogenous plasma biotin levels ${ }^{24}$. A probe variant with yet greater biotin sensitivity can be generated by reducing the number of desthiobiotinylation sites, although this results in a substantially lower dynamic range (Supplementary Fig. 5). The response of BT-AVATar to biotin is reversible over repeated cycles of excess SA and biotin addition (Fig. 3b).

The purpose of a biotin sensor is to map biotin-labeled targets in vivo, analogous to a variety of biochemical and histological techniques, but in intact tissue. To test functionality of BTAVATar for this purpose, we used the PAC1 bioassay to evaluate the sensor's in vitro responses to biotin moieties immobilized on molecules and cells (Fig. 3c, Supplementary Fig. 6). As expected, BT-AVATar is strongly activated by both biotinylated bovine serum albumin and HEK293 cells, exhibiting increases in PAC1 receptor activation by $675 \pm 69 \%$ and $227 \pm 30 \%$, respectively, versus minimal responses to unbiotinylated controls (Fig. 3d). These results show that BT-AVATar maintains sensitivity and selectivity for biotin even in biological environments, suggesting the suitability of vasoprobe-based sensors for applications in vivo.

Ultrasensitive AVATar-based molecular imaging in vivo. To demonstrate the ability of BT-AVATar to enable sensitive molecular imaging of biotinylated targets in live animals, we implanted xenografts of $\sim 500,000$ biotinylated or nonbiotinylated cells each at symmetric sites in rat cortex and performed molecular imaging using the probe (Fig. 4a). In order to survey a large region of the brain with minimal invasiveness, including but not limited to the cell implantation sites, BT-AVATar was infused distally into the cerebrospinal fluid (CSF) at the rostral extent of the cortex. Dissemination of imaging agents introduced using this route was verified by injecting the conventional MRI contrast agent gadolinium diethylenetriaminepentaacetic acid (Gd-DTPA) and then visualizing the results by $T_{1}$-weighted imaging. Although BT-AVATar and Gd-DTPA have different predicted diffusion coefficients, the spread of these agents is expected to be dominated by convection ${ }^{25}$ and therefore well represented by gadolinium-dependent contrast enhancement in our experiments. An infusion of $40 \mu \mathrm{L}$ of $200 \mathrm{mM}$ Gd-DTPA spread throughout the anterior quadrant of the brain, enhancing contrast across a large volume including the cell implantation site, as well as additional regions of the olfactory bulb, frontal cortex, prelimbic cortex, motor cortex, and cingulate cortex (Fig. 4b).

In the area of the implanted cells, BT-AVATar produces MRI signal changes with amplitudes that clearly distinguish biotinylated from control xenografts (Fig. 4c). Mean hemodynamic 
a

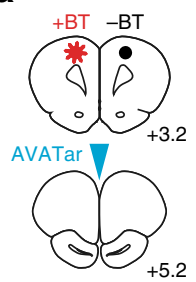

C

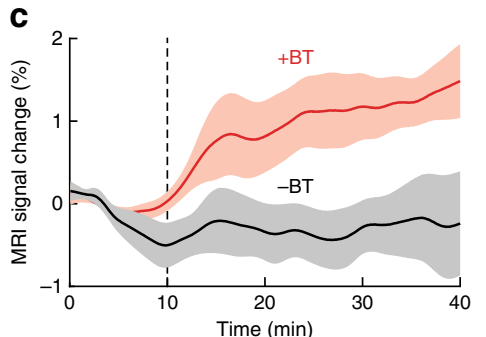

b

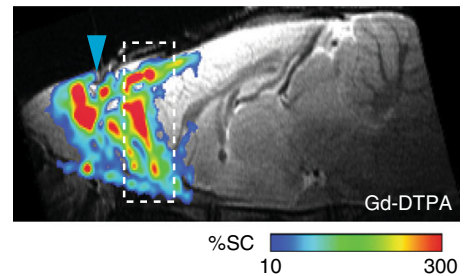

d

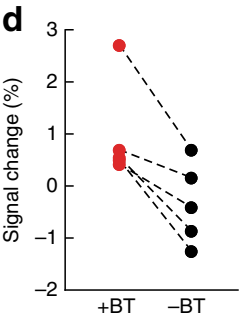

e

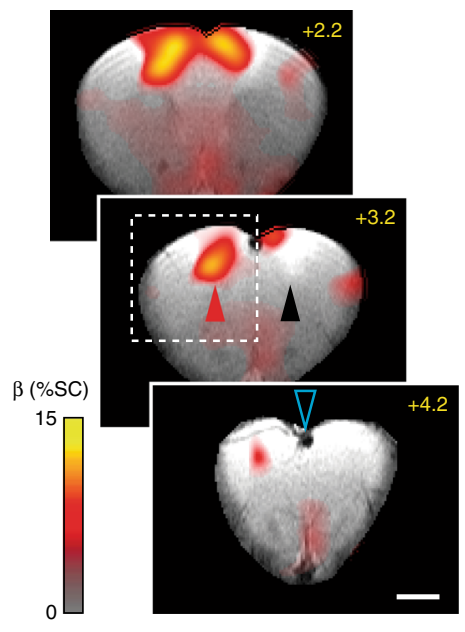

f
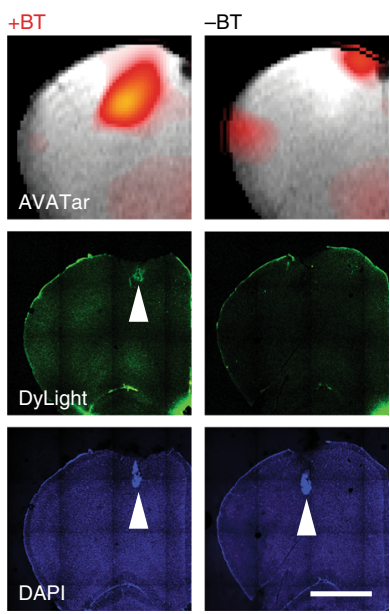

Fig. 4 In vivo molecular imaging with BT-AVATar. a Brain atlas ${ }^{57}$ sections (bregma coordinates noted) showing positions of test ( $+B T$, red) and control (-BT, black) cell implants and intra-CSF AVATar infusion for in vivo mapping experiments. b Wide-field probe delivery illustrated using Gd-DTPA infused at the AVATar infusion position (blue arrowhead). Color scale illustrates signal changes (\%SC) produced by the contrast agent. c Signal change over time near test $(+B T$, red) and control ( $-B T$, black) cells. Dashed line indicates start of AVATar infusion. Shading denotes SEM of $n=5$. d Signal enhancements in individual animals at $+B$ T (red) and $-B T$ (black) sites. e Map of regression coefficients ( $\beta$, in units of \%SC) illustrating responses near $+B$ T cell implant locations (red arrowhead) but not -BT controls (black arrowhead). BT-AVATar was infused 1 mm anterior to the open blue arrowhead. Displayed brain slices correspond to the dashed region in $\mathbf{b}$. Scale bar $=2 \mathrm{~mm}$. $\mathbf{f}$ Comparison of MRI results with histology shows overlap of BT-AVATar signal with position of test implants (left images) but not control implants (right images). Top row: closeup of the dashed region in e (left), containing $+B T$ cells, juxtaposed with the symmetric region containing -BT cells (right). Middle row: selective activation of BT-AVATar by biotinylated cells is confirmed by visualization of biotinylated cells (left, arrowhead) but not unbiotinylated cells (right) following infusion of fluorescent BT-AVATar formulated with DyLight-labeled SA. Bottom row: visualization of both test and control xenografts (arrowheads) by DAPI staining. Scale bar $=2$ mm.

responses average $1.0 \pm 0.4 \%$ in the neighborhood of biotinylated cells, versus $-0.3 \pm 0.4 \%$ near control cells (Fig. $4 \mathrm{~d}$ ), a significant difference (paired $t$-test $p=0.01, n=5$ ). Temporal analysis of the wide-field imaging data produces a map of corresponding changes over a wide field of view; this map indicates signal changes clustered primarily near the area of biotinylated cell implantation, despite wide-field administration of BT-AVATar (Fig. 4e). Comparison of the molecular imaging map with histological results confirms colocalization of the AVATarmediated signal with biotin-containing xenografts (Fig. 4f). Estimation of biotin content in the test cell implants indicates that 0.4 pmol biotin was present in each xenograft, corresponding to concentrations of about $150 \mathrm{nM}$ if cells are undiluted following injection (Supplementary Fig. 7). Based on the error margins of results presented in Fig. 4d, this appears to be about two-fold greater than the limit of biotin detection in vivo under the imaging conditions we used. This biotin concentration is also far below analyte levels detectable by conventional paramagnetic MRI contrast agents. These results demonstrate that nanomolar target concentrations can be imaged using BT-AVATar in conjunction with wide-field CSF delivery methods in rat brain.

AVATar-based neurotransmitter detection. The AVATar design can be generalized for sensing a wide variety of molecular targets. Neurochemicals are of particular interest because of their distinct functional roles in the nervous system and their importance in health and disease. Although neurotransmitterresponsive probes for MRI have been reported, they are not sensitive enough to report the submicromolar extracellular concentrations characteristic of many species. To adapt the sensing mechanism of BT-AVATar for neurotransmitter detection, we mutated PACAP residues to accommodate conjugation to synthetic thiol-reactive derivatives of dopamine (DA), a neurochemical involved in addiction, motivated behavior, and motor control (Fig. 5a, Supplementary Figs. 8 and 9). A PACAP conjugate modified with DA at positions 10 and 20 showed an $\mathrm{EC}_{50}$ value of $2.5 \pm 0.6 \mathrm{nM}$ for PAC1 receptor activation in the cellbased assay (Fig. 5b), indicating that tolerance for modification of PACAP at these residues is general. The dopamine sensor DAAVATar was formed by mixing the modified peptide with excess immunoglobulin $\mathrm{G}$ directed against the tethered neurotransmitter moieties. In this sensor, the DA-specific IgG functions as a blocking domain according to the design of Fig. 1b, analogous to the SA component in BT-AVATar, with blocking reversed upon addition of excess DA (Fig. 5c).

Titration of DA-AVATar with DA indicates that the sensor has an $\mathrm{EC}_{50}$ value of $5.6 \pm 0.5 \mu \mathrm{M}$ for its analyte, with a limit of detection below $1 \mu \mathrm{M}$ (Fig. $5 \mathrm{~d}$ ). Simple modeling of the DAAVATar mechanism indicates that its neurotransmitter sensitivity could be altered by changing the number of DA conjugation sites on PACAP, as well as the affinity of the IgG for both tethered and free DA (Supplementary Fig. 10). In keeping with these predictions, we found that a triply dopaminylated PACAP could be used to assemble a DA-AVATar with higher $\mathrm{EC}_{50}$ but enhanced dynamic range (Supplementary Fig. 11). These results therefore demonstrate that the AVATar principle can be used to create tunable probes with high sensitivity for a variety of small molecules.

\section{Discussion}

Our work thus introduces AVATars as a versatile nonmagnetic and radiation-free imaging probe architecture for detecting molecular targets with nanomolar sensitivity in deep tissue. These probes can be applied at concentrations more than 1,000-fold 

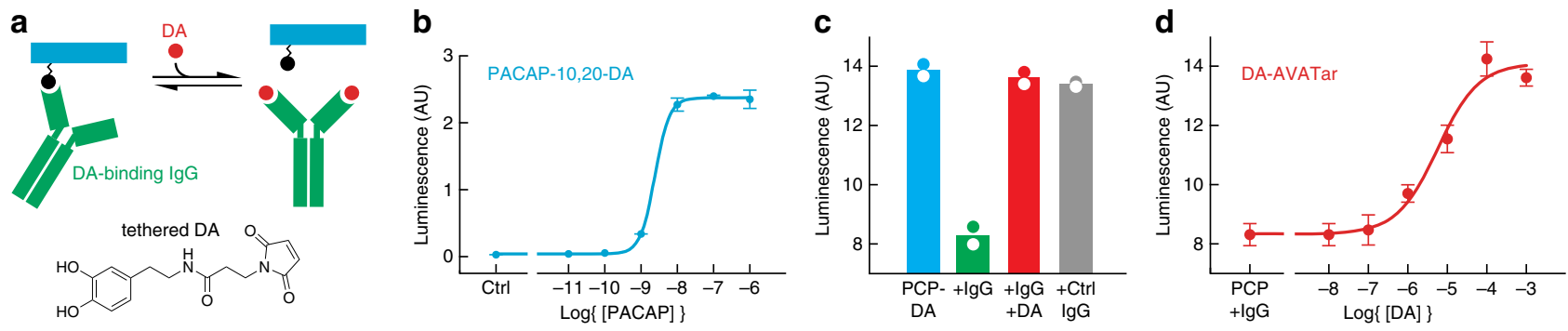

Fig. 5 A neurotransmitter-sensitive AVATar. a Mechanism of dopamine-sensitive AVATars: PACAP (blue) is modified with a tethered dopamine (DA) analog (black), and a DA-binding lgG (green) functions as the blocking domain. Free neurotransmitter (red) competes with this interaction, releasing active PACAP. Structure of thiol-reactive tethered DA shown at bottom. $\mathbf{b}$ Titration of PACAP-10,20-DA in the PAC1 cell assay, showing efficient receptor activation. c PAC1 receptor activation by $1 \mathrm{nM}$ PACAP-10,20-DA in the absence (blue) or presence (green) of $600 \mathrm{nM}$ DA-binding lgG. Activity is restored by addition of excess DA (red). Control lgG (gray) does not block PACAP activity. $\mathbf{d}$ Titration of the DA response of $1 \mathrm{nM}$ DA-AVATar in the PAC1 activation assay. DA activates DA-AVATar with an $\mathrm{EC}_{50}$ of $5.6 \pm 0.5 \mu \mathrm{M}$. Error bars denote SD of duplicates.

lower than conventional paramagnetic MRI agents, comparable to some nuclear imaging probes, making them particularly suitable for noninvasive imaging of the many biological species that are present at submicromolar concentrations in vivo. We designed the biotin-sensitive imaging agent BT-AVATar, which is able to visualize biotin-labeled cell populations following widefield delivery of the probe in live rat brains. We also demonstrate adaptability of the AVATar architecture by designing an agent that detects the neurotransmitter DA. Incorporation of alternative tethered ligands and cognate binding domains could permit a variety of further vasoprobe-based sensors to be constructed, in some cases leveraging well-characterized components of previously described fluorescent imaging probes ${ }^{26-35}$.

Both BT-AVATar and DA-AVATar display sensitivity to submicromolar levels of their targets. Such high analyte sensitivity can also be achieved using nanoparticle-based MRI contrast agents ${ }^{36,37}$, but these agents are often difficult to deliver broadly in tissue, due to their large size. Paramagnetic agents generally need to be applied at concentrations above $10 \mu \mathrm{M}$, making detection of nanomolar-scale targets difficult for stoichiometric reasons. Using BT-AVATar, we demonstrated that $150 \mathrm{nM}$ biotin present in cell implants can be detected in vivo. Although the xenografts we imaged contained $\sim 0.5$ million cells each, similar levels of biotin could be used to label far fewer cells or other species of interest. For instance, BT-AVATar could be used for direct detection of targets biotinylated in vivo ${ }^{38}$, or for indirect detection in conjunction with pretargeted antibody-biotin conjugates, in both cases assuming realistic levels of target expression and biotinylation. We did not apply the neurotransmitter-sensitive DA-AVATar probe in vivo, but the probe's in vitro sensitivity probably makes it suitable for noninvasive imaging of low micromolar DA release levels evoked by behaviorally rewarding stimuli ${ }^{39}$. Compared with paramagnetic MRI-detectable DA sensors, DAAVATar could be applied at sensor concentrations three orders of magnitude lower, which are unlikely to perturb endogenous neurochemical function. In contrast to other MRI agents, AVATars are also compatible with detection by any hemodynamic imaging modality9.

The high sensitivity of vasoprobe-based agents for their targets arises from their exploitation of physiological amplification pathways built into the vasculature. Quantification of analyte levels using this approach should be possible in contexts where calibration of AVATar-mediated signals can be performed with respect to known target concentrations. The PAC1 activation assay used here could provide an external standard for this. For instance, if we suppose that the biotin-triggered BT-AVATar responses of Fig. 4 constitute about half the maximum response of $\sim 25 \%$ observed in similar hemodynamic imaging experiments, then we would crudely but correctly infer that the amount of biotin detected approximately matches the in vitro $\mathrm{EC}_{50}$ of $\mathrm{BT}$ AVATar for biotin. This external calibration approach could be improved by performing explicit measurements of maximal hemodynamic changes in situ, using established techniques ${ }^{40-42}$.

The spatiotemporal properties of AVATar-based analyte sensing likewise follow largely from their unique hemodynamic mechanism. The probes are compatible with diverse hemodynamic imaging methods, including magnetic, nuclear, ultrasonic, and optical approaches that in some cases provide spatial detail on the order of single blood vessels $(<100 \mu \mathrm{m})$. The temporal resolution provided by AVATars is likely to be limited by hemodynamic response time courses on the order of seconds, and may be further limited by the kinetics of the competitive interactions between analytes and the blocking domains used in the sensor designs presented here. For neurotransmitter sensing, kinetic characteristics of AVATars may thus be comparable to previous neurotransmitter-sensitive MRI probes, which display response time constants of tens of seconds; these are favorable with respect to receptor displacement strategies used in positron emission tomography, which generally require measurements lasting tens of minutes.

The probe architecture we describe here entails the combination of $\sim 5 \mathrm{kDa}$ PACAP derivatives with proteins that range from 60 to $150 \mathrm{kDa}$ in molecular weight. Even the largest of these has a hydrodynamic radius smaller than most nanoparticle-based imaging agents. The relatively small size and high potency of AVATars should facilitate delivery of these probes to a number of tissue types in which they are predicted to elicit analyte-dependent hemodynamic responses ${ }^{43-48}$. The brain is of particular interest because of its important chemical signaling systems and inaccessibility to most diagnostic agents. Interestingly, PACAP derivatives have been shown in earlier studies to spontaneously permeate the blood-brain barrier ${ }^{49-52}$, suggesting the possibility of completely noninvasive delivery of PACAP-based sensors to the central nervous system. Although this avenue remains to be explored, the experiments presented here already demonstrate that AVATars can access large fields of view in the rodent brain following minimally invasive intra-CSF infusion. Similar probes applied using such delivery approaches could thus provide unprecedented capability for monitoring molecular targets throughout much of the body.

\section{Methods}

Plasmids. Lentiviral plasmids were constructed using the Golden Gate method ${ }^{53}$ in the pLentiX1 Zeo plasmid (Addgene \#17299) with its kanamycin resistance replaced by the ampicillin resistance cassette. The Glo22F gene encoding an cAMPmodulated luciferase (Promega, Madison, WI) ${ }^{54}$ was cloned followed by an internal ribosome entry site, a Cerulean fluorescent protein, a $2 \mathrm{~A}$ viral sequence $\mathrm{e}^{55}$ and a puromycin resistance marker. The PAC1 gene was cloned followed by an internal 
ribosome entry site, an mKate fluorescent protein, a $2 \mathrm{~A}$ viral sequence and a blasticidin resistance marker. Lentiviral helper plasmids pMD2.G (Addgene \#12259, Cambridge, MA) and psPAX2 (Addgene \#12260) were gifts from Didier Trono.

Mammalian cell culture. CHO K1 cells were purchased from Sigma-Aldrich (St. Louis, MO) and cultured in 90\% F10 medium supplemented with 10\% fetal bovine serum (FBS), 100 units $/ \mathrm{mL}$ penicillin, and $100 \mu \mathrm{g} / \mathrm{mL}$ streptomycin. Cells were frozen in freezing medium composed of $80 \%$ F10 medium, $10 \%$ FBS and $10 \%$ dimethylsulfoxide.

\begin{abstract}
Lentivirus production and cell line generation. 293FT cells (Thermo Fisher Scientific, Waltham, MA) were seeded into six-well plates at 1 million cells per well and transfected using Lipofectamine 2000 (Life Technologies, Grand Island, NY) according to instructions at sub-confluence. Co-transfection of $0.5 \mathrm{mg}$ pMD2.G, $1 \mathrm{mg}$ psPAX 2 and $1 \mathrm{mg}$ of the lentiviral plasmid of interest was performed with $6.25 \mathrm{ml}$ Lipofectamine 2000 reagent. Virus-containing supernatant was collected after 48 and $72 \mathrm{~h}$, filtered through $0.45 \mathrm{~mm}$ filters, and used for infection. Super natants were stored at $4{ }^{\circ} \mathrm{C}$ for up to a week. CHO K1 cells were seeded into 24 -well plates at 40,000 cells per well in the presence of $4 \mathrm{mg} / \mathrm{ml}$ Polybrene in $50 \%$ fresh medium and $50 \%$ viral supernatants containing both Glo22F and PAC1 viruses. The medium was replaced with fresh viral supernatants daily for 4 days. Selection was performed using both antibiotic resistance and fluorescent markers for each lentivirus. Beginning on day 3 after initial infection, $10 \mathrm{mg} / \mathrm{ml}$ blasticidin and $1 \mathrm{mg} / \mathrm{ml}$ puromycin (Life Technologies) were added to the medium for selection and selection was continued until all cells expressed the appropriate fluorescent markers.
\end{abstract}

PAC1 luminescence assay. Production of intracellular cAMP mediated by activation of the PACAP receptor PAC1 was measured using the GloSensor assay ${ }^{54}$ (Promega, Madison, WI). We generated a $\mathrm{CHO} \mathrm{K} 1$ cell line expressing the required cAMP-dependent luciferase and PAC1 receptor. $24 \mathrm{~h}$ before the assay, 2500 cells per well were seeded in $100 \mu \mathrm{F} 10$ with $10 \%$ FBS in white opaque clear-bottom 96well plates (Costar, Coppell, TX). Before the assay, the medium was removed from the wells and replaced with $90 \mu \mathrm{l}$ per well of Gibco $\mathrm{CO}_{2}$-independent medium (Life Technologies) with $10 \%$ FBS containing $1 \% \mathrm{v} / \mathrm{v}$ of cAMP GloSensor substrate stock solution (Promega). The cells were incubated in substrate-containing medium at $37^{\circ} \mathrm{C}$ in $5 \% \mathrm{CO}_{2}$ for $2-4 \mathrm{~h}$ and equilibrated to room temperature and atmospheric $\mathrm{CO}_{2}$ for $30 \mathrm{~min}$. All samples were prepared as duplicates in standard phosphatebuffered saline (PBS), $\mathrm{pH} 7.4$, plus $0.1 \%(\mathrm{w} / \mathrm{v})$ 3-[(3-cholamidopropyl)dimethylammonio]-1-propanesulfonate (CHAPS) to reduce adsorptive loss of free peptides. $10 \mu \mathrm{L}$ of ten times the desired final concentration were added per well. Luminescence was measured for $30 \mathrm{~min}$ using a microtiter plate reader and evaluated after the signal reached a plateau $(15 \mathrm{~min})$. Dose-response curves were fitted to a four-parameter Hill equation using Prism (GraphPad Software, San Diego, CA). Additional data analysis was performed using proFit (QuantumSoft, Uetikon am See, Switzerland). $\mathrm{EC}_{50}$ values are reported as mean and standard deviation of duplicate measurements.

Preparation and in vitro testing of AVATars. All peptides were produced by solid-state synthesis at the MIT Koch Institute Biopolymers and Proteomics Laboratory and purified by high-performance liquid chromatography (HPLC). Biotin (BT) and desthiobiotin (dBT) were incorporated as lysine sidechainderivatized monomers in the synthesis; polyethylene glycol (PEG)-linked BT was incorporated as a BT-functionalized glutamine monomer spaced using a linker of four PEG units. Identity and purity of each peptide was confirmed by matrixassisted laser desorption ionization (MALDI)-time of flight mass spectrometry and analytical HPLC. After lyophilization, the peptides were weighed, dissolved in water to a stock concentration of $1 \mathrm{mM}$ and stored at $-80^{\circ} \mathrm{C}$. PAC1 receptor activation by PACAP derived probes was tested with the luminescence assay described above. Activity of PACAP conjugates to BT or dBT were assessed in absence and presence of streptavidin (SA; Thermo Fisher Scientific). For sensor activation studies, BT-AVATars were formed by premixing $1 \mathrm{nM}$ PACAP-dBT conjugates with a two-fold molar excess of SA and subsequently activated by addition of BT. Reversibility of this sensing was probed by successively adding the following reagents to $1 \mathrm{nM}$ PACAP-dBT: $2 \mathrm{nM}$ SA, $80 \mathrm{nM}$ biotin, $200 \mathrm{nM} \mathrm{SA}, 8 \mu \mathrm{M}$ biotin. Further BT-AVATar activation studies were performed using biotinylated bovine serum albumin (BSA, Sigma-Aldrich) containing $100 \mathrm{nM}$ BT or biotinylated cells containing $150 \mathrm{nM}$ BT (preparation see below) as analytes.

To prepare dopamine (DA)-sensitive AVATar, DA was functionalized with a maleimido moiety to provide a facile and thiol-selective conjugation with PACAP substituted at positions 10 and 20 with cysteine residues (PACAP-10,20-Cys). Addition of the maleimido functional group was achieved by reacting DA with $\mathrm{N}$ succinimidyl 3-maleimidopropionate in anhydrous dimethylformamide and further purification using HPLC. The resulting functionalized neurotransmitter, DA-Mal, was further characterized by standard methods (Supplementary Methods and Supplementary Figs. 8 and 9). DA-Mal was then reacted with PACAP-10,20Cys in PBS pH 7.4 using a 6-fold excess of DA-Mal. The resulting conjugate, PACAP-10,20-DA, was purified by HPLC and its molecular structure was confirmed by high-resolution MALDI mass spectrometry. The DA-AVATar was formed by premixing $1 \mathrm{nM}$ PACAP-10,20-DA with $600 \mathrm{nM}$ anti-dopamine IgG antibody (ab6427; Abcam, Cambridge, MA). Dopamine sensitivity of the DAAVATar was examined using freshly prepared DA (Sigma-Aldrich) stocks in PBS $\mathrm{pH} 7.4$.

Computational modeling of DA-AVATar design parameters. Dependence of DA-AVATar performance on the number of PACAP-tethered DA moieties and $K_{d}$ values for tethered and free DA was modeled using a custom script running in MATLAB. The model assumed that IgG domains bind tethered PACAP-tethered DA moieties independently and with equal affinity, that these interactions are in competition with free DA binding to the IgG domains, and that the concentration of DA is not depleted by binding. Model parameters included: a total PACAP concentration of $10 \mathrm{nM}$, DA concentrations ranging from 0 to $100 \mu \mathrm{M}$, a $K_{d}$ for IgG binding to tethered DA of $10 \mathrm{nM}$, a number of tethered DA moieties $(n)$ per peptide from 1 to 3 , a concentration of blocking IgG domains equal to the total concentration of tethered DA moieities (i.e. 10,20 , or $30 \mathrm{nM}$ ), and $K_{d}$ values for IgG binding to free DA of $10 \mathrm{nM}, 100 \mathrm{nM}$, or $1 \mu \mathrm{M}$. Under each set of parameters, the relative activity of the DA-AVATar was computed as the fraction of unblocked PACAP conjugate expected under equilibrium conditions, equal to $U T^{n}$, where $U T$ is the fraction of unbound PACAP-tethered DA.

Preparation of biotinylated cell xenografts. HEK293 Freestyle cells were separated into two $10 \mathrm{~mL}$ samples 30 million cells/sample. The cells were washed twice with $10 \mathrm{~mL}$ ice cold PBS pH 7.4 and resuspended in $1 \mathrm{~mL}$ each. The test sample was biotinylated by adding $120 \mu \mathrm{L}$ of biotinylation reagent (EZ-link NHS-Biotin, Thermo Fisher Scientific). In all, $120 \mu \mathrm{L}$ of PBS pH 7.4 was added to the control cells. The cells were nutated for $30 \mathrm{~min}$ at room temperature and another $30 \mathrm{~min}$ at $4{ }^{\circ} \mathrm{C}$. They were then washed three times with ice cold PBS, $\mathrm{pH} 7.4$, and resuspended in $100 \mu \mathrm{L}$ artificial cerebrospinal fluid (aCSF). Biotinylation of the cell surface was quantified by labeling biotinylated and control cells with fluorescent SA (SA-DyLight488, Thermo Fisher Scientific). Cells were diluted 1:5 in PBS pH 7.4 and $1 / 10$ of the volume was incubated with $0.1 \mathrm{mg} \mathrm{SA}$-DyLight 488 for $30 \mathrm{~min}$ at room temperature. Afterwards, cells were washed three times with $\mathrm{PBS} \mathrm{pH} 7.4$ to remove unreacted SAand diluted 1:10. DyLight488 fluorescence was quantified using a microtiter plate reader (Excitation: $485 \mathrm{~nm}$, Emission: $538 \mathrm{~nm}$ ). Cell surface biotinylation was estimated by comparing SA-DyLight fluorescence of cells to a SA-DyLight 488 dilution series. All samples were prepared in duplicate. These procedures were performed immediately before implantation experiments described below.

Animal procedures. All animal procedures were conducted in accordance with National Institutes of Health guidelines and with the approval of the MIT Committee on Animal Care (protocol number 0718-068-21). All experiments were performed with male Sprague-Dawley rats, age 7-9 weeks, supplied by Charles River Laboratories (Wilmington, MA). Seventeen rats were used for in vivo imaging experiments described here.

Preparation of animals for PACAP injection. Seven rats underwent surgery to implant bilateral cannula guides over the caudate putamen region of the striatum $(\mathrm{CPu})$. Anesthesia was induced using 3\% isoflurane and maintained using $2 \%$ isoflurane with vacuum suction turned on to remove excess anesthetic. The rats were injected subcutaneously with $1.2 \mathrm{mg} / \mathrm{kg}$ of sustained release buprenorphine for analgesia. Eyes were covered with Paralube Vet Ointment (Dechra Veterinary Products, Overland Park, KS) to prevent drying from exposure to isoflurane. The animals' heads were then shaved and cleaned with alcohol and povidone-iodine prep pads for easy access to the skull. Using sterile surgical equipment, the skin over the skull was retracted and the skull cleaned of tissue so that the sutures on the skull were clearly visible. Holes were drilled through the skull for the cannula guides bilaterally at $3 \mathrm{~mm}$ lateral to midline, $0.5 \mathrm{~mm}$ anterior from bregma. A small 26 gauge needle was used to puncture the dura in each of the drilled holes, allowing smooth access to the brain parenchyma. The holes were air dried and bilaterally connected custom-made cannula guides (22 gauge PEEK, $6 \mathrm{~mm}$ distance between the guides, Plastics One, Roanoke, VA) were inserted into the holes, such that they protruded $1 \mathrm{~mm}$ into the brain parenchyma. The guides were then secured to the skull using white dental cement (C\&B Metabond, Parkell). Next, a custom head post was attached to the rats' skulls posterior to the cannula guide implantation sites using the white dental cement. After the cement dried, pink cold cure dental cement (Teets Denture Material, Patterson Dental, Saint Paul, MN) was applied over the white cement to secure the entire implant. Tissue glue was then applied to seal the surface connecting the cement and skin areas around the implant. Cannula guides were sealed with dummy cannulas (protruding as far as the guide) to avoid exposure of brain tissue during the recovery period.

MRI assessment of injected PACAP probes. Seven days after the implantation of the bilateral cannulas, the rats were imaged during PACAP intracranial infusion. For the imaging experiments, animals were anesthetized using $2 \%$ isoflurane in oxygen for induction. After numbing the trachea with lidocaine, animals were intubated intratracheally using the 16 gauge plastic part of a Surflash I.V. catheter (Terumo Medical Products, Somerset, NJ). The rats were then connected to a small 
animal respirator (Inspira Advanced Safety Ventilator; Harvard Apparatus, Holliston, MA), and fixed via their headposts into a custom-built cradle for imaging with a commercial surface radiofrequency coil for MRI detection (Doty Scientific, Columbia, SC) fitting snugly around the headpost. Breathing rate and end-tidal expired $\mathrm{CO}_{2}$ were continuously monitored. Once animals were positioned in the cradle, the anesthesia was lowered to $0.75 \%$ isoflurane and the rats were paralyzed with pancuronium ( $1 \mathrm{mg} / \mathrm{kg}$ IP bolus for induction, $2 \mathrm{mg} / \mathrm{kg} / \mathrm{h} \mathrm{IP}$ infusion) to prevent motion artifacts during imaging.

Immediately before each experiment, two injection cannulas, designed to protrude $4 \mathrm{~mm}$ into the brain past the cannula guides reaching into the $\mathrm{CPu}(28$ gauge PEEK internal cannula, Plastics One, Roanoke, VA, USA), were attached to $25 \mu \mathrm{L}$ Hamilton glass syringes and prefilled with the appropriate intracranial injection solution (aCSF or $1 \mu \mathrm{M}$ PACAP in aCSF). Injection cannulas were then lowered, while applying positive pressure of $0.01 \mu \mathrm{L} / \mathrm{min}$ to avoid air bubbles, into the previously implanted bilateral cannula guides. Next, the Hamilton syringes were placed in a remote infuse/withdraw dual syringe pump (PHD 2000 Syringe Pump; Harvard Apparatus). A birdcage resonator coil for radiofrequency excitation in MRI (Doty Scientific) was positioned around the cradle, and the ensemble was inserted into the MRI magnet bore and locked in a position such that the head of the animal was at the center of the bore.

Animals were scanned by MRI using a $20 \mathrm{~cm}$-horizontal bore $7 \mathrm{~T}$ or $9.4 \mathrm{~T}$ scanner (Bruker BioSpin, Billerica, MA) to measure the changes in hemodynamic contrast following intracranial injections. High resolution $T_{2}$-weighted anatomical scans of each animal were obtained using a rapid acquisition with relaxation enhancement (RARE) pulse sequence with echo time (TE) $44 \mathrm{~ms}$, recycle time (TR) $2,500 \mathrm{~ms}$, RARE factor 8, spatial resolution $100 \mu \mathrm{m} \times 100 \mu \mathrm{m} \times 1 \mathrm{~mm}$, and matrix size $256 \times$ 256 with seven slices. Hemodynamic contrast image series were acquired using a gradient echo planar imaging (EPI) pulse sequence with TE $25 \mathrm{~ms}$, TR 2,000 ms, spatial resolution $390 \mu \mathrm{m} \times 390 \mu \mathrm{m} \times 1 \mathrm{~mm}$, and matrix size $64 \times 64$ with seven slices. Ten minutes of baseline measurement with $4 \mathrm{~s}$ per time point (i.e. averaging two $T R=2 \mathrm{~s}$ EPI scans per image) were acquired before probe infusion. Following the baseline period, while continuously collecting EPI scans, the infusion pump was remotely turned on to commence intracranial injection of $1 \mu \mathrm{M}$ PACAP or control solutions at the rate of $0.1 \mu \mathrm{L} / \mathrm{min}$ for $10 \mathrm{~min}$ through the cannulas. EPI scans were collected for an additional 20 min after the infusion of the PACAP probe.

MRI data were processed and analyzed using the AFNI software package ${ }^{56}$. The AFNI 3dAllineate command was used to align each animal's EPI data set to the corresponding RARE anatomical image. Each animal's image data were then aligned to the cannula tip of a reference anatomical MRI scan. Data from each voxel were normalized to 100 , based on the mean of the baseline time period, using AFNI's 3dcalc command. To identify voxels with significant increases or decreases in BOLD signal, we compared the signal observed $10 \mathrm{~min}$ after the infusion to the baseline in the group data. For visualization, group signal change maps were overlaid on a reference anatomical image. Time courses were obtained by averaging MRI signal over $1.2 \times 1.2 \mathrm{~mm}$ regions of interest defined around cannula tip locations in individual animals' datasets and standard error was calculated across animals using MATLAB. The plateau percent signal change was determined by comparing values observed during the baseline period with values observed after PACAP infusion (30-40 min).

Preparation of animals for imaging biotin-labeled targets. Five rats underwent surgery for implantation of biotinylated and control cells approximately three hours prior to imaging. Craniotomies were performed as described above, except that for these experiments, three holes were drilled through the skull: one for intraCSF injection of AVATars on the midline just behind the olfactory bulbs $(5.2 \mathrm{~mm}$ anterior from the bregma), and two for bilateral cell implantations $2 \mathrm{~mm}$ lateral to midline and $3.2 \mathrm{~mm}$ anterior from bregma. A 1-mm-long guide cannula was implanted at the midline site. To introduce the cells at the lateral sites, two metal cannulas (33 gauge, Plastics One) chosen to minimize damage caused to the brain tissue were attached to $25 \mu \mathrm{L}$ Hamilton glass syringes and prefilled with the freshly biotinylated or control HEK cells. The injection cannulas were then lowered $2 \mathrm{~mm}$ into the brain parenchyma through the two lateral craniotomies and $3 \mu \mathrm{L}$ of cell suspension ( $\sim 0.5$ million cells) was injected over $30 \mathrm{~min}$ at $0.1 \mu \mathrm{L} / \mathrm{min}$. After the cell injection was completed, the metal cannulas were removed, the cell injection holes were dried and sealed, headpost attachment was performed, and a dummy cannula was applied to each cannula guide, all as described above.

In vivo imaging with BT-AVATar. Approximately $2 \mathrm{~h}$ after cell implantation, animals were further prepared for imaging and examined in conjunction with wide-field BT-AVATar infusion. The rats were again intubated, ventilated, and positioned in the MRI cradle with surface coil before being taken off of isoflurane and placed on $0.05 \mathrm{mg} / \mathrm{mL}$ dexmedetomidine anesthesia mixed with $1 \mathrm{mg} / \mathrm{mL}$ pancuronium for paralysis $(1 \mathrm{~mL} / \mathrm{kg}$ IP bolus for induction, $2 \mathrm{~mL} / \mathrm{kg} / \mathrm{h}$ IP infusion afterwards). An internal cannula designed to protrude $0.25 \mathrm{~mm}$ past the preimplanted midline cannula guide was attached to $50 \mu \mathrm{L}$ Hamilton glass syringe and prefilled with BT-AVATar $(2 \mu \mathrm{M}$ PACAP-10,20,32-dBT mixed with $20 \mu \mathrm{M} \mathrm{SA})$. The injection cannula was then lowered into the guide, followed by placement of the volume coil over the animal and insertion into the scanner as described above. Animals were scanned by MRI using the same parameters used to validate PACAP itself. Following a ten minute baseline period, infusion of BT-AVATar into the CSF was initiated at a rate of $1 \mu \mathrm{L} / \mathrm{min}$ during continuous scanning.

Data were again processed and analyzed using AFNI. After alignment of each animal's EPI data to the corresponding anatomical RARE scan, the datasets were all registered in order to align their cell injection sites to those of a reference animal. To eliminate the contribution of nonspecific image fluctuations, we normalized each animal's time course data to the time course of a reference region in the most posterior slice, assumed not to be affected by AVATar infusion. We then scaled the baseline intensity of each voxel to 100 using AFNI's 3 dcalc command. We excluded from analysis voxels displaying $\sim 50 \%$ or greater hypointensity prior to AVATar infusion, which we took to indicate tissue damage in the area of cell injections. To identify voxels with signal changes indicative of AVATar activation, we applied AFNI's $3 \mathrm{dDeconvolve}$ command to identify signal components that displayed a linear increase during AVATar infusion followed by a plateau thereafter. Motion parameters were used as nuisance regressors. For visualization, the resulting beta coefficient maps (in units of percent change) were overlaid on a reference anatomical image. Time courses were obtained by averaging MRI signal from $2.5 \times$ $2.5 \mathrm{~mm}$ regions of interest defined around the centroid of cell implantation in individual animals, as judged from anatomical scans. Mean and standard error were calculated across animals using MATLAB. The percent signal change in individual animals was determined by comparing signal values observed during baseline and infusion conditions (15-30 $\mathrm{min})$.

\section{MRI of BOLD response evoked by somatosensory stimulation. A rat was} anesthetized and intubated as described for in vivo imaging with BT-AVATar. The animal was then ventilated and transferred to the MRI cradle. Subcutaneous electrodes were inserted in the left forepaw and then connected to a stimulus isolator. MRI images were obtained on a $9.4 \mathrm{~T}$ scanner (Bruker). A transmit-only $70 \mathrm{~cm}$ inner diameter linear volume coil (Bruker) and a $2 \mathrm{~cm}$ diameter receive-only surface coil (Doty Scientific) were used for excitation and detection, respectively. Scanner operation was performed using the Paravision 6.0.1 software (Bruker). High resolution anatomical MRI images were acquired using a $T_{2}$-weighted RARE pulse sequence with RARE factor $=8$, effective $T E=30 \mathrm{~ms}, T R=5 \mathrm{~s}$, in-plane $\mathrm{FOV}=1.92 \times 1.92 \mathrm{~cm}$, in-plane resolution $100 \times 100 \mu \mathrm{m}$, and slice thickness $=$ $1 \mathrm{~mm}$ for six coronal slices.

Functional imaging with left forepaw stimulation consisted of six blocks of $3 \mathrm{~mA} 1 \mathrm{~ms}$ pulses repeated at $9 \mathrm{~Hz}$ for $10 \mathrm{~s}$ epochs alternating with $40 \mathrm{~s}$ of rest. In each case, the stimulation paradigm was synchronized with the image acquisition using a custom script executed in LabVIEW (National Instruments, Austin, TX). To minimize potential radiofrequency artifacts, the stimulator cable was filtered with a $60 \mathrm{MHz}$ low-pass filter (Mini-Circuits, Brooklyn, NY) before entering the MRI enclosure. An EPI sequence was used for detection of stimulus-induced BOLD contrast, with $T E=20 \mathrm{~ms}, T R=2 \mathrm{~s}, \mathrm{FOV}=2.56 \times 2.56 \mathrm{~cm}$, in-plane resolution $400 \times 400 \mu \mathrm{m}$, and slice thickness $=1 \mathrm{~mm}$. The same precise imaging sequence was also used to evaluate the MRI signals arising from injected PACAP probes at $9.4 \mathrm{~T}$ this procedure was also applied with $T R=1 \mathrm{~s}$ to compare PACAP-induced signals obtained at different temporal resolutions.

EPI images were processed using AFNI. Motion correction and alignment of EPI images to the corresponding anatomical RARE scan were performed in the same way as for in vivo imaging with BT-AVATar. Stimulus-dependent time courses for each voxel were estimated using the $3 \mathrm{dDeconvolve} \mathrm{general} \mathrm{linear}$ modeling implementation in AFNI. Left forepaw stimulus time blocks were used as event regressors. Six motion correction parameters were included as nuisance regressors, along with a linear baseline term. Outlier scans detected by median deviation from time series trends in each data set were censored from the analysis BOLD response amplitude map was computed in MATLAB as the mean signal for $10 \mathrm{~s}$ (five image frames) beginning at stimulus onset, minus the baseline signal defined by the mean of response at time points $1-20 \mathrm{~s}$ (10 frames) before stimulus onset.

\section{Assessment of contrast agent spread upon intra-CSF infusion. Ability of} molecular imaging probes to spread broadly through the brain during intra-CSF injection was confirmed using test infusions of gadolinium diethylenetriaminepentaacetic acid (Gd-DTPA, Sigma-Aldrich). In each animal, an intra-CSF cannula guide and headpost were implanted and animals were prepared for imaging as described above, except that the intra-CSF infusion syringe was filled instead with $200 \mathrm{mM}$ Gd-DTPA formulated in aCSF. After insertion of animals into the scanner and acquisition of anatomical scans, $T_{1}$-weighted scans suitable for visualizing Gd-DTPA effects were performed using a fast low-angle shot (FLASH) pulse sequence with TE $5 \mathrm{~ms}$, TR $93.75 \mathrm{~ms}$, spatial resolution $400 \mu \mathrm{m} \times 400 \mu \mathrm{m} \times$ $1 \mathrm{~mm}$, and matrix size $64 \times 64$ with seven sagittal slices. Scans were collected over a ten minute baseline period followed by $30 \mathrm{~min}$ of contrast agent infusion at a rate of $1 \mu \mathrm{L} / \mathrm{min}$. The resulting MRI data were processed and analyzed using AFNI. To identify voxels with significant increases or decreases in $T_{1}$ signal, we compared the signal during the final ten minutes of Gd-DTPA infusion to the baseline signal from each animal and expressed the result as a percent change in mean intensity. For visualization, a representative signal change map was overlaid on the corresponding sagittal anatomical image. 
Histology. Fluorescent BT-AVATar was prepared by formulating the probes with SA-DyLight 488 (Thermo Fisher Scientific) in place of unlabeled SA. Surgical preparation and biotin-labeled cell implantation were then performed as described above. After infusion procedures identical to those used in the MRI experiments, animals were transcardially perfused with PBS followed by $4 \%$ paraformaldehyde in PBS. Brains were extracted, post-fixed overnight at $4{ }^{\circ} \mathrm{C}$, and sectioned the following day. Free-floating sections $(50 \mu \mathrm{m})$ were cut using a vibratome (VT1200 S, Leica Microsystems GmbH, Wetzlar, Germany), mounted on glass slides with ProLong Gold Antifade Mountant (Thermo Fisher Scientific) and protected with a coverslip. BT-AVATar activation in the area of the cell implantation was visualized by the green fluorescence arising from SA-DyLight 488. Independent of biotinylation, the distribution of xenografted cells was also visualized by staining with DAPI (1:1000 dilution from a $1 \mathrm{mg} / \mathrm{mL}$ stock, Thermo Fisher Scientific), followed by a wash with PBS plus $0.5 \%$ BSA. Fluorescence imaging was performed using a confocal microscope (Axio Imager 2, Zeiss, Thornwood, NY).

Reporting summary. Further information on research design is available in the Nature Research Reporting Summary linked to this article.

\section{Data availability}

Source data are provided as supplementary information. Additional data unsuitable for deposition are available from the corresponding author upon reasonable request.

\section{Code availability}

No major code was developed for this study. Minor custom routines used in data analysis are specific to this work, and are available from the corresponding author upon reasonable request.

Received: 14 August 2019; Accepted: 3 April 2020;

Published online: 13 May 2020

\section{References}

1. Lin, M. Z. \& Schnitzer, M. J. Genetically encoded indicators of neuronal activity. Nat. Neurosci. 19, 1142-1153 (2016)

2. Wang, H., Jing, M. \& Li, Y. Lighting up the brain: genetically encoded fluorescent sensors for imaging neurotransmitters and neuromodulators. Curr. Opin. Neurobiol. 50, 171-178 (2018).

3. Rice, W. L., Shcherbakova, D. M., Verkhusha, V. V. \& Kumar, A. T. N. In vivo tomographic imaging of deep-seated cancer using fluorescence lifetime contrast. Cancer Res. 75, 1236-1243 (2015).

4. Ji, N., Freeman, J. \& Smith, S. L. Technologies for imaging neural activity in large volumes. Nat. Neurosci. 19, 1154-1164 (2016).

5. Flusberg, B. A. et al. Fiber-optic fluorescence imaging. Nat. Methods 2, 941-950 (2005).

6. Ouzounov, D. G. et al. In vivo three-photon imaging of activity of GCaMP6labeled neurons deep in intact mouse brain. Nat. Methods 14, 388-390 (2017).

7. Phelps, M. E. Positron emission tomography provides molecular imaging of biological processes. Proc. Natl Acad. Sci. USA 97, 9226-9233 (2000).

8. Ghosh, S., Harvey, P., Simon, J. C. \& Jasanoff, A. Probing the brain with molecular fMRI. Curr. Opin. Neurobiol. 50, 201-210 (2018).

9. Desai, M., Slusarczyk, A. L., Chapin, A., Barch, M. \& Jasanoff, A. Molecular imaging with engineered physiology. Nat. Commun. 7, 13607 (2016).

10. Wintermark, $M$. et al. Comparative overview of brain perfusion imaging techniques. J. Neuroradiol. 32, 294-314 (2005).

11. Hillman, E. M. C. Optical brain imaging in vivo: techniques and applications from animal to man. J. Biomed. Opt. 12, 051402 (2007).

12. Buxton, R. B. The physics of functional magnetic resonance imaging (fMRI). Rep. Prog. Phys. 76, 096601 (2013).

13. Deffieux, T., Demene, C., Pernot, M. \& Tanter, M. Functional ultrasound neuroimaging: a review of the preclinical and clinical state of the art. Curr Opin. Neurobiol. 50, 128-135 (2018).

14. Robberecht, P. et al. Structural requirements for the occupancy of pituitary adenylate-cyclase-activating-peptide (PACAP) receptors and adenylate cyclase activation in human neuroblastoma NB-OK-1 cell membranes. Eur. J. Biochem. 207, 239-246 (1992).

15. Wilchek, M. \& Bayer, E. A. Methods Enzymol. 184, 5-13 (1990).

16. Altai, M., Membreno, R., Cook, B., Tolmachev, V. \& Zeglis, B. M. Pretargeted imaging and therapy. J. Nucl. Med. 58, 1553-1559 (2017).

17. Green, N. M. Avidin. 3. The nature of the biotin-binding site. Biochem. J. 89, 599-609 (1963).

18. Sun, C. et al. Solution structure and mutational analysis of pituitary adenylate cyclase-activating polypeptide binding to the extracellular domain of PAC1RS. Proc. Natl. Acad. Sci. U.S.A. 104, 7875-7880 (2007).
19. Vaudry, D. et al. Pituitary adenylate cyclase-activating polypeptide and its receptors: 20 years after the discovery. Pharmacol. Rev. 61, 283-357 (2009).

20. Gourlet, P. et al. Structural requirements for the binding of the pituitary adenylate-cyclase-activating peptide to receptors and adenylate-cyclase activation in pancreatic and neuronal membranes. Eur. J. Biochem. 195, 535-541 (1991).

21. Klumb, L. A., Chu, V. \& Stayton, P. S. Energetic roles of hydrogen bonds at the ureido oxygen binding pocket in the streptavidin-biotin complex. Biochemistry 37, 7657-7663 (1998).

22. Hofmann, K., Titus, G., Montibeller, J. A. \& Finn, F. M. Avidin binding of carboxyl-substituted biotin and analogues. Biochemistry 21, 978-984 (1982).

23. Hirsch, J. D. et al. Easily reversible desthiobiotin binding to streptavidin, avidin, and other biotin-binding proteins: uses for protein labeling, detection, and isolation. Anal. Biochem. 308, 343-357 (2002).

24. Clevidence, B. A., Marshall, M. W. \& Canary, J. J. Biotin levels in plasma and urine of healthy adults consuming physiological doses of biotin. Nutr. Res. 8 , 1109-1118 (1988)

25. Hai, A., Cai, L. X., Lee, T., Lelyveld, V. S. \& Jasanoff, A. Molecular fMRI of Serotonin Transport. Neuron 92, 754-765 (2016).

26. Looger, L. L. et al. A genetically encoded fluorescent sensor for in vivo imaging of GABA. Nat. Methods 16, 763-770 (2018).

27. De Lorimier, R. M. et al. Construction of a fluorescent biosensor family. Protein Sci. 11, 2655-2675 (2002).

28. Marvin, J. S., Schreiter, E. R., Echevarría, I. M. \& Looger, L. L. A genetically encoded, high-signal-to-noise maltose sensor. Proteins 79, 3025-3036 (2011)

29. Marvin, J. S. et al. An optimized fluorescent probe for visualizing glutamate neurotransmission. Nat. Methods 10, 162-170 (2013).

30. Okumoto, S. et al. Detection of glutamate release from neurons by genetically encoded surface-displayed FRET nanosensors. Proc. Natl Acad. Sci. U.S.A. 102, 8740-8745 (2005).

31. Brun, M. A. et al. A semisynthetic fluorescent sensor protein for glutamate. $J$ Am. Chem. Soc. 134, 7676-7678 (2012).

32. Brun, M. A. et al. Semisynthesis of fluorescent metabolite sensors on cell surfaces. J. Am. Chem. Soc. 133, 16235-16242 (2011)

33. Griss, R. et al. Bioluminescent sensor proteins for point-of-care therapeutic drug monitoring. Nat. Chem. Biol. 10, 598-603 (2014).

34. Masharina, A., Reymond, L., Maurel, D., Umezawa, K. \& Johnsson, K. A fluorescent sensor for GABA and synthetic $\mathrm{GABA}_{\mathrm{B}}$ receptor ligands. J. Am. Chem. Soc. 134, 19026-19034 (2012).

35. Schena, A. \& Johnsson, K. Sensing acetylcholine and anticholinesterase compounds. Angew. Chem. Int. Ed. Eng. 53, 1302-1305 (2014).

36. Perez, J. M., Josephson, L. \& Weissleder, R. Use of magnetic nanoparticles as nanosensors to probe for molecular interactions. ChemBioChem 5, 261-264 (2004).

37. Hsieh, V. et al. Neurotransmitter-responsive nanosensors for $\mathrm{T}_{2}$-weighted magnetic resonance imaging. J. Am. Chem. Soc. 141, 15751-15754 (2019).

38. Bartelle, B. B. et al. Novel genetic approach for in vivo vascular imaging in mice. Circ. Res. 110, 938-947 (2012).

39. Lee, T., Cai, L. X., Lelyveld, V. S., Hai, A. \& Jasanoff, A. Molecular-level functional magnetic resonance imaging of dopaminergic signaling. Science 344, 533-535 (2014).

40. Davis, T. L., Kwong, K. K., Weisskoff, R. M. \& Rosen, B. R. Calibrated functional MRI: mapping the dynamics of oxidative metabolism. Proc. Natl Acad. Sci. U.S.A. 95, 1834-1839 (1998).

41. Hoge, R. D. et al. Investigation of BOLD signal dependence on cerebral blood flow and oxygen consumption: the deoxyhemoglobin dilution model. Magn. Reson. Med. 42, 849-863 (1999).

42. Germuska, M. \& Wise, R. G. Calibrated fMRI for mapping absolute $\mathrm{CMRO}_{2}$ : Practicalities and prospects. NeuroImage 187, 145-153 (2019).

43. Stalder, A. F. et al. Robust cardiac BOLD MRI using an fMRI-like approach with repeated stress paradigms. Magn. Reson. Med. 73, 577-585 (2015).

44. Haque, M., Koktzoglou, I., Li, W., Carbray, J. \& Prasad, P. Functional MRI of liver using BOLD MRI: effect of glucose. J. Magn. Reson. Imaging JMRI 32, 988-991 (2010).

45. Jacobi, B. et al. Skeletal muscle BOLD MRI: from underlying physiological concepts to its usefulness in clinical conditions. J. Magn. Reson. Imaging 35, 1253-1265 (2012)

46. Hall, M. E., Jordan, J. H., Juncos, L. A., Hundley, W. G. \& Hall, J. E. BOLD magnetic resonance imaging in nephrology. Int. J. Nephrol. Renov. Dis. 11, 103-112 (2018).

47. Ogawa, S., Lee, T. M., Kay, A. R. \& Tank, D. W. Brain magnetic resonance imaging with contrast dependent on blood oxygenation. Proc. Natl. Acad. Sci. U.S.A. 87, 9868-9872 (1990).

48. Buchbinder, B. R. Functional magnetic resonance imaging. Handb. Clin. Neurol. 135, 61-92 (2016)

49. Banks, W. A., Kastin, A. J., Komaki, G. \& Arimura, A. Passage of pituitary adenylate cyclase activating polypeptide1-27 and pituitary adenylate cyclase activating polypeptide1-38 across the blood-brain barrier. J. Pharmacol. Exp. Ther. 267, 690-696 (1993). 
50. Banks, W. A., Uchida, D., Arimura, A., Somogyvári-Vigh, A. \& Shioda, S. Transport of pituitary adenylate cyclase-activating polypeptide across the blood-brain barrier and the prevention of ischemia-induced death of hippocampal neurons. Ann. N. Y. Acad. Sci. 805, 270-277 (1996).

51. Dogrukol-Ak, D., Tore, F. \& Tuncel, N. Passage of VIP/PACAP/secretin family across the blood-brain barrier: therapeutic effects. Curr. Pharm. Des. 10, 1325-1340 (2004).

52. Chatenet, D., Fournier, A. \& Bourgault, S. PACAP-Derived Carriers: Mechanisms and Applications. in Pituitary Adenylate Cyclase Activating Polypeptide - PACAP, Vol. 11 (eds Reglodi, D. \& Tamas, A.) 133-148 (Springer International Publishing, 2016).

53. Engler, C., Kandzia, R. \& Marillonnet, S. A one pot, one step, precision cloning method with high throughput capability. PLoS ONE 3, e3647 (2008).

54. Fan, F. et al. Novel genetically encoded biosensors using firefly luciferase. ACS Chem. Biol. 3, 346-351 (2008).

55. Szymczak, A. L. et al. Correction of multi-gene deficiency in vivo using a single 'self-cleaving' $2 \mathrm{~A}$ peptide-based retroviral vector. Nat. Biotechnol. 22, 589 (2004).

56. Cox, R. W. AFNI: software for analysis and visualization of functional magnetic resonance neuroimages. Comput. Biomed. Res. Int. J. 29, 162-173 (1996).

57. Paxinos, G. \& Watson, C. The Rat Brain in Stereotaxic Coordinates - 6th edn. (Academic Press, 2007).

\section{Acknowledgements}

We acknowledge grants from the National Institutes of Health (R24 MH109081, U01 NS103470, and UF1 NS107712) to A.J. R.O. was funded by a fellowship from the Deutsche Forschungsgemeinschaft. A.W. was funded by the Advanced Multimodal Neuroimaging Training Program at the Massachusetts General Hospital (R90 DA023427) and a fellowship from Harvard-MIT Health Sciences and Technology program. We thank Dr. Peter Harvey for help with fluorescence microscopy, and are grateful to Alla Leshinsky of the MIT Koch Institute Biopolymers and Proteomics Laboratory for assistance with peptide synthesis.

\section{Author contributions}

R.O performed the principal in vitro experiments. A.W. performed the principal in vivo experiments. R.O., A.S., and A.J. designed the AVATars. A.W., M.D., and A.J. designed the experimental approaches. A.B. designed and synthesized tethered dopamine. N.L. performed the comparison of vasoprobe and BOLD fMRI results. R.O., A.W., and A.J. wrote the paper with assistance from other authors.

\section{Competing interests}

The authors declare no competing interests.

\section{Additional information}

Supplementary information is available for this paper at https://doi.org/10.1038/s41467 020-16118-7.

Correspondence and requests for materials should be addressed to A.J.

Peer review information Nature Communications thanks Albrecht Stroh, Eva Jakab Toth and the other, anonymous, reviewer for their contribution to the peer review of this work.

Reprints and permission information is available at http://www.nature.com/reprints

Publisher's note Springer Nature remains neutral with regard to jurisdictional claims in published maps and institutional affiliations.

(c) (i) Open Access This article is licensed under a Creative Commons Attribution 4.0 International License, which permits use, sharing, adaptation, distribution and reproduction in any medium or format, as long as you give appropriate credit to the original author(s) and the source, provide a link to the Creative Commons license, and indicate if changes were made. The images or other third party material in this article are included in the article's Creative Commons license, unless indicated otherwise in a credit line to the material. If material is not included in the article's Creative Commons license and your intended use is not permitted by statutory regulation or exceeds the permitted use, you will need to obtain permission directly from the copyright holder. To view a copy of this license, visit http://creativecommons.org/ licenses/by/4.0/.

(C) The Author(s) 2020 